\title{
Commentary
}

\section{Cardiovascular disease registers and recording of behavioural risk factors: why untapped opportunities continue}

Diet and physical activity are of immense importance to primary and secondary prevention of coronary heart disease $(\mathrm{CHD})^{1}$. However, information relating to these activities at the individual and population level remains scant, with opportunities to alter the situation overlooked continuously. A recent independent review in the UK was highly critical of existing information systems to assess needs and plan service provision ${ }^{2}$. Others are critical too in relation to $\mathrm{CHD}$ specifically ${ }^{3}$. We argue that the existing poor information infrastructure relating to diet, physical activity and other variables associated with a person's way of life, known to influence CHD, could be improved by utilising disease registers. We put forward key arguments why this may not be happening and encourage practitioners and researchers to help overcome such barriers.

Disease-specific registers are increasingly being developed in countries such as the UK in response to national quality frameworks ${ }^{4,5}$. General practitioners in the UK are given financial incentives to establish administrative systems to identify patients with and at significant risk for CHD, with the purpose of facilitating the systematic delivery of care and regular patient follow-up. Earlier work suggests that disease-specific registers can provide good-quality information relating to individual patients as well as for monitoring and service planning ${ }^{6-8}$. It has been argued that well-administered CHD registers enable patient profiling and patterns of care to be examined within individuals or groups of general practices ${ }^{9,10}$. Yet it remains to be seen if $\mathrm{CHD}$ and other registers lead to enhanced patient care ${ }^{11}$.

The possible benefits of CHD registers extend beyond individual patient care and that delivered by a single care provider. Advances in technology make it feasible to develop single registers within a particular administrative district, for example, with primary and secondary care providers linking 'real-time' information, regardless of their place of work. Not only would this allow patterns of care to be monitored between providers and over time, it would also create a unified longitudinal dataset with which to examine the often complex relationships between patient and clinical/treatment variables and changes in outcomes over time. This analysis can be missing from clinical trials because of short follow-up times, focus on a single primary outcome measure, or because trial participants are not always representative of the general population. Information on variations in inputs and their effect on patient care also provides analysis from 'natural experiments', again argued to be an untapped resource ${ }^{2}$.

Concerns remain about using data from CHD registers for research purposes, alongside missed opportunities for individual patient care. Many CHD registers focus on optimal prescription of CHD drugs, which are underpinned by national government quality indicators. However, these indicators provide no incentive to adequately record variables associated with $\mathrm{CHD}$ and a person's way of life, which is usually limited to assessing smoking behaviour. This is at odds with the evidence base showing a strong impact of diet and physical activity on the total population burden of $\mathrm{CHD}^{1}$ and with guidelines for preventing and treating this disease $\mathrm{e}^{12,13}$.

Thus clinicians are currently denied systematic methods for recording and reviewing patient information relating to diet and physical activity during a patient consultation, despite its potential contribution to reducing their risk of future events or their recovery following an event. Why are clinicians expected to measure and record blood pressures over time in patients with or at risk of CHD, yet not do the same for diet and physical activity? This absence of information also poses problems to the research community. Failing to measure and account for potential confounding variables, of which diet and physical activity are, will seriously (and rightly so) reduce the validity of research findings based on CHD registry data. It is, in effect, as serious as a longitudinal analysis of $\beta$-blockers ignoring blood pressure in its analysis.

\section{Why does the problem remain?}

If diet and physical activity are so important to the primary and secondary prevention of $\mathrm{CHD}$, then why does this information remain absent from CHD registers? We put forward two related arguments. The first rests with clinicians. We suggest that some clinicians, following a clinically dominated training, remain unaware of the true strength of the impact of diet and physical activity on the course of CHD. While attention will probably be given to cholesterol for example, this still fails to consider wider dietary components and their impact on CHD. 
This scenario is perhaps reinforced through pharmaceutical marketing techniques, diverting attention from interventions to change a patient's way of life where this impacts on their illness. Others may feel lacking in skills to address such issues, and some could be daunted by what they see as an impossible task in supporting appropriate dietary changes and increases in physical activity by their patients.

A second but related issue refers to the availability (or lack) of suitable methods or instruments to measure diet and physical activity systematically within the constraints of a general practice consultation and then to record this easily on the patient register. Few simple instruments exist to measure dietary intake, with most designed to examine nutrient-disease relationships. We argue this level of detail is not necessary within the context of CHD registers. However, information would be of value to clinicians, service planners and researchers alike if it identified the extent that patients followed key dietary recommendations in relation to CHD protection. Short-form tools are available to assess physical activity, although not validated in this context, but still remain absent from CHD and other disease registers ${ }^{14}$.

We accept that complex issues need to be resolved in developing a short instrument to collect and record this type of information within the confines of a patient consultation. However, it is not outwith such possibilities ${ }^{15}$ although existing tools are not suitable for this task ${ }^{16}$. We do not accept that complexity is a good enough argument to continue to ignore diet and physical activity in CHD registers, given their importance in the primary and secondary prevention of CHD. No doubt many sceptics prevailed when short-form mental health instruments were mooted. Yet now a number of short instruments are available to collect robust information on dimensions of mental health and are used successfully in patient consultations ${ }^{17}$.

CHD registers offer hope in improving individual patient care and in addressing local and national information gaps about this dominant chronic disease. However, registers that continue to ignore the contribution of diet and physical activity to the primary and secondary prevention of CHD will only reinforce traditional healthcare practice, and do nothing to encourage clinicians to pay attention to patients' wider way of life and its impact on their illness. Moreover, doubt will remain about the validity of research findings from CHD registers. This diminishes the potential of the registers as tools of population health surveillance until the effect confounding from diet and physical activity can be assessed alongside other routinely collected data. The challenge to the research community is to now develop such shortform assessment methods, and to clinicians, to incorporate these as part of the patient consultation. It then remains to be seen if a CHD register, capable of prompting and storing all information appropriate to the care of individual patients, leads to significant improvements in outcomes over time. Without this, the cost and effort involved in managing any register is futile.

\section{Roger A Harrison}

Directorate of Public Health, Bolton Primary Care Trust

Bolton, UK and

Evidence for Population Health Unit

Room 2.909 Stopford Building

University of Manchester

Oxford Road, Manchester M13 9PT, UK

Email: roger.harrison-2@manchester.ac.uk

Georgios Lyratzopoulos

Evidence for Population Health Unit

University of Manchester

Manchester, UK

\section{References}

1 McPherson K, Britton A, Causer L. Coronary Heart Disease: Estimating the Impact of Changes in Risk Factors. London: The Stationery Office, 2002.

2 Wanless D. Securing the Health of the Whole Population. Final Report. London: HMSO, 2004.

3 Unal B, Critchley JA, Capewell S. Missing, mediocre, or merely obsolete? An evaluation of UK data sources for coronary heart disease. Journal of Epidemiology and Community Health 2003; 57: 530-5.

4 British Medical Association. The New GMS Contract: Investing in General Medical Services [online], 2003 Available at http://www.bma.org.uk/ap.nsf/Content/investinggp. Accessed 10 April 2004

5 Department of Health. National Service Framework for Coronary Heart Disease. London: Department of Health, 2000.

6 Carroll K, Majeed A, Firth C, Gray J. Prevalence and management of coronary heart disease in primary care: population-based cross-sectional study using a disease register. Journal of Public Health Medicine 2003; 25: 29-35.

7 Gray D, Hampton JR. Twenty years' experience of myocardial infarction: the value of a heart attack register. British Journal of Clinical Practice 1993; 47: 292-5.

8 Pierce M, Lundy S, Palanisamy A, Winning S, King J. Prospective randomised controlled trial of methods of call and recall for cervical cytology screening. British Medical Journal 1989; 299: 160-2.

9 Pringle M, Ward P, Chilvers C. Assessment of the completeness and accuracy of computer medical records in four practices committed to recording data on computer. British Journal of General Practice 1995; 45: 537-41.

10 Whitelaw FG, Taylor RJ, Nevin SL, Taylor MW, Milne RM, Watt AH. Completeness and accuracy of morbidity and repeat prescribing records held on general practice computers in Scotland. British Journal of General Practice 1996; 46: 181-6.

11 Moher M, Yudkin P, Wright L, Turner R, Fuller A, Schofield T, et al. Cluster randomised controlled trial to compare three methods of promoting secondary prevention of coronary heart disease in primary care. British Medical Journal 2001; 322: $1338-42$.

12 Grundy SM, Balady GJ, Criqui MH, Fletcher G, Greenland P, Hiratzka LF, et al. Primary prevention of coronary heart disease: guidance from Framingham. A statement for healthcare professionals from the AHA task force on risk reduction. Circulation 1998; 97: 1876-87. 
13 National Institute for Clinical Excellence. Audit of the Management of Post-MI Patients in Primary Care [online]. National Collaborating Centre for Primary Care, 2001. Available at http://www.nice.org.uk/pdf/postmiaudit.pdf. Accessed 10 April 2004.

14 Miller DJ, Freedson PS, Kline GM. Comparison of activity levels using Caltrac accelerometer and five questionnaires. Medicine and Science in Sports and Exercise 1994; 26: $376-82$.

15 Little P, Barnett J, Margetts B, Kinmonth AL, Gabbay J,
Thompson R, et al. The validity of dietary assessment in general practice. Journal of Epidemiology and Community Health 1999; 53: 165-6.

16 Little P, Margetts B. Dietary and exercise assessment in general practice. Family Practice 1996; 13: 477-82.

17 Hickie IB, Andrews G, Davenport TA. Measuring outcomes in patients with depression or anxiety: an essential part of clinical practice. Medical Journal of Australia 2002; 177: $205-7$. 